\title{
Genetic diversity and delineation of Salmonella Agona outbreak strains by next generation sequencing, Bavaria, Germany, 1993 to 2018
}

Alexandra Dangel ${ }^{1,2}$, Anja Berger ${ }^{1,2}$, Ute Messelhäußer ${ }^{1}$, Regina Konrad ${ }^{1}$, Stefan Hörmansdorfer ${ }^{1}$, Nikolaus Ackermann¹, Andreas Sing 1

1. Bavarian Health and Food Safety Authority (LGL), Oberschleissheim, Germany

2. These authors contributed equally to this article

Correspondence: Alexandra Dangel (alexandra.dangel@lgl.bayern.de)

Citation style for this article:

Dangel Alexandra, Berger Anja, Messelhäußer Ute, Konrad Regina, Hörmansdorfer Stefan, Ackermann Nikolaus, Sing Andreas. Genetic diversity and delineation of Salmonella Agona outbreak strains by next generation sequencing, Bavaria, Germany, 1993 to 2018. Euro Surveill. 2019;24(18):pii=1800303. https://doi. org/10.2807/1560-7917.ES.2019.24.18.1800303

Article submitted on 11 Jun 2018 / accepted on 31 Dec 2018 / published on 02 May 2019

Background: In 2017, a food-borne Salmonella Agona outbreak caused by infant milk products from a French supplier occurred in Europe. Simultaneously, S. Agona was detected in animal feed samples in Bavaria. Aim: Using next generation sequencing (NGS) and three data analysis methods, this study's objectives were to verify clonality of the Bavarian feed strains, rule out their connection to the outbreak, explore the genetic diversity of Bavarian S. Agona isolates from 1993 to 2018 and compare the analysis approaches employed, for practicality and ability to delineate outbreaks caused by the genetically monomorphic Agona serovar. Methods: In this observational retrospective study, three 2017 Bavarian feed isolates were compared to a French outbreak isolate and $48 \mathrm{~S}$. Agona isolates from our strain collections. The later included human, food, feed, veterinary and environmental isolates, of which 28 were epidemiologically outbreak related. All isolates were subjected to NGS and analysed by: (i) a publicly available species-specific core genome multilocus sequence typing (cgMLST) scheme, (ii) single nucleotide polymorphism phylogeny and (iii) an in-house serovar-specific cgMLST scheme. Using additional international S. Agona outbreak NGS data, the cluster resolution capacity of the two cgMLST schemes was assessed. Results: We could prove clonality of the feed isolates and exclude their relation to the French outbreak. All approaches confirmed former Bavarian epidemiological clusters. Conclusion: Even for $S$. Agona, species-level cgMLST can produce reasonable resolution, being standardisable by public health laboratories. For single samples or homogeneous sample sets, higher resolution by serovar-specific cgMLST or SNP genotyping can facilitate outbreak investigations.

\section{Introduction}

Salmonellosis is one of the most common food-borne human diseases. It is often transmitted via contaminated meat, eggs or seafood products. Moreover, due to the robustness of Salmonella spp., dried products like herbs or spices have also proven their potential as vehicle of infection. Of more than 2,600 different serovars of $S$. enterica, only a few non-typhoidal serovars are responsible for most human infections. In S. enterica subsp. enterica, these serovars include for example Enteridis, Typhimurium and Agona. In Europe, S. Agona is far from leading the list of pathogenic serovars, as cases of $S$. Enteritidis and $S$. Typhimurium are much more numerous [1]. Globally, S. Agona is a common pathogen and food-borne outbreaks connected to it have been consistently reported in several countries. Examples are a 2002-03 outbreak, caused by aniseedfennel-caraway tea products affecting 77 patients in Germany [2,3], a 2008 outbreak connected to meat products of one supplier causing 163 infections in 10 different countries with most cases in the United Kingdom [4], a 2011 multi-state outbreak in the United States (US) caused by fresh papaya resulting in more than 100 infected patients [5], or a point-sourced outbreak caused by tuna sushi in Sydney, Australia in 2015 [6].

In outbreak investigations, serotyping and phage typing have been used for decades in many laboratories including reference laboratories. Serotyping is still serving as a gold-standard technique for routine typing. In combination with other typing techniques like phage typing, it may be suited for the investigation of small, geographically limited outbreaks [7]. However, many serovars are polyphyletic and serotyping sometimes confounds genetically unrelated isolates and thus does not recognise evolutionary groupings in some cases. Therefore, attempts were made some 
TABLE 1A

Characteristics of sequenced Bavarian Salmonella Agona isolates and French representative outbreak isolate, Germany, $1993-2018(n=52)$

\begin{tabular}{|c|c|c|c|c|c|c|c|c|}
\hline Sample name & Year & Material & $\begin{array}{l}\text { Isolation } \\
\text { source }\end{array}$ & Country/state & $\begin{array}{l}\text { Country of } \\
\text { origin/travel } \\
\text { history }\end{array}$ & $\begin{array}{l}\text { S. enterica } \\
\text { cgMLST CT }\end{array}$ & $\begin{array}{l}\text { cgMLST } \\
\text { cluster }\end{array}$ & $\begin{array}{l}\text { Epidemiological } \\
\text { link }\end{array}$ \\
\hline$E^{2} R 2219379^{a}$ & 2017 & NA & Human & France & NA & 704 & ND & NA \\
\hline SAooo1 & 2011 & Stool & Human & Germany/Bavaria & Iraq & 1195 & 2 & Travel cluster \\
\hline SA0002 & 2011 & Stool & Human & Germany/Bavaria & Iraq & 1215 & ND & NA \\
\hline SA0004 & 2012 & Stool & Human & Germany/Bavaria & Afghanistan & 1195 & 2 & Travel cluster \\
\hline SA0005 & 2012 & Stool & Human & Germany/Bavaria & Madagascar & 1209 & None & NA \\
\hline SA0006 & 2013 & Stool & Human & Germany/Bavaria & Germany & 1210 & ND & NA \\
\hline SA0007 & 2013 & Stool & Human & Germany/Bavaria & Russia & 1211 & ND & NA \\
\hline SA0008 & 2013 & Bacterial strain & Human & Germany/Bavaria & Germany & 1212 & ND & NA \\
\hline SA0009 & 2013 & Bacterial strain & Human & Germany/Bavaria & Germany & 1199 & 6 & NA \\
\hline SAoo11 & 2014 & Stool & Human & Germany/Bavaria & $\begin{array}{l}\text { Unspecified } \\
\text { foreign } \\
\text { country }\end{array}$ & 1214 & 8 & NA \\
\hline SA0012 & 2014 & Stool & Human & Germany/Bavaria & Syria & 1195 & 2 & Travel cluster \\
\hline SA0013 & 2014 & Stool & Human & Germany/Bavaria & Syria & 1195 & 2 & Travel cluster \\
\hline SA0015 & 2014 & Stool & Human & Germany/Bavaria & Syria & 1194 & ND & NA \\
\hline SA0016 & 2014 & Stool & Human & Germany/Bavaria & Nigeria & 1195 & 2 & Travel cluster \\
\hline SAo017 & 2015 & Stool & Human & Germany/Bavaria & $\begin{array}{c}\text { Kosovo } \\
\text { under UN } \\
\text { Security } \\
\text { Council } \\
\text { Resolution } \\
1244\end{array}$ & 1196 & ND & NA \\
\hline SA0018b & 2015 & Stool & Human & Germany/Bavaria & Eritrea & 1214 & $8^{b}$ & NA \\
\hline SA0019 & 2015 & Bacterial strain & Human & Germany/Bavaria & Colombia & 1197 & ND & NA \\
\hline $\mathrm{SA} 0031^{\mathrm{b}}$ & 2018 & $\begin{array}{l}\text { Nutritional } \\
\text { supplement }\end{array}$ & $\begin{array}{l}\text { Nutritional } \\
\text { supplement }\end{array}$ & Germany/Bavaria & NA & 1513 & $N D^{b}$ & NA \\
\hline SA0032 & 2017 & Stool & Human & Germany/Bavaria & NA & 1198 & ND & NA \\
\hline SA0033 & 2017 & Stool & Human & Germany/Bavaria & NA & 1124 & ND & NA \\
\hline SA0034 & 2017 & Stool & Human & Germany/Bavaria & NA & 1199 & 6 & NA \\
\hline SA0035 & 2017 & Cattle & Cattle & Germany/Bavaria & NA & 1200 & ND & NA \\
\hline SAoo36 & 2015 & Chicken faeces & Environment & Germany/Bavaria & NA & 1134 & 4 & Chicken cluster \\
\hline SA0037 $7^{b, c}$ & 2015 & Cattle & Cattle & Germany/Bavaria & NA & NDC & $\mathrm{ND}^{\mathrm{b}, \mathrm{c}}$ & NA \\
\hline SAoo38 & 2015 & Chicken faeces & Environment & Germany/Bavaria & NA & 1134 & 4 & Chicken cluster \\
\hline SA0039 & 2015 & Chicken faeces & Environment & Germany/Bavaria & NA & 1134 & 4 & Chicken cluster \\
\hline SAo0 40 & 2015 & Chicken & Environment & Germany/Bavaria & NA & 1201 & ND & NA \\
\hline SAoo 41 & 2008 & Black pepper & Spices & Germany/Bavaria & NA & 1202 & ND & NA \\
\hline SA0042 & 2017 & Animal feed & Animal feed & Germany/Bavaria & NA & 1193 & 3 & Feed cluster \\
\hline SAoo 43 & 2017 & Animal feed & Animal feed & Germany/Bavaria & NA & 1193 & 3 & Feed cluster \\
\hline SAo0 44 & 2017 & Animal feed & Animal feed & Germany/Bavaria & NA & 1193 & 3 & Feed cluster \\
\hline SAoo 45 & 2003 & Digestive tea & Tea/raw tea & Germany/Bavaria & NA & 1203 & 1 & Tea outbreak \\
\hline SAo046 & 2003 & Digestive tea & Tea/raw tea & Germany/Bavaria & NA & 1203 & 1 & Tea outbreak \\
\hline SA0047 & 2003 & $\begin{array}{c}\text { Cough and } \\
\text { bronchial tea }\end{array}$ & Tea/raw tea & Germany/Bavaria & NA & 1203 & 1 & Tea outbreak \\
\hline SAo0 48 & 1994 & Turkey leg & Food & Germany/Bavaria & NA & 1204 & ND & NA \\
\hline SA0050 & 1994 & $\begin{array}{c}\text { Shredded } \\
\text { coconut }\end{array}$ & Food & Germany/Bavaria & NA & 1205 & 5 & Coconut cluster \\
\hline SAoo 51 & 1994 & $\begin{array}{c}\text { Shredded } \\
\text { coconut }\end{array}$ & Food & Germany/Bavaria & NA & 1207 & 7 & NA \\
\hline SA0052 & 1994 & $\begin{array}{l}\text { Shredded } \\
\text { coconut }\end{array}$ & Food & Germany/Bavaria & NA & 1205 & 5 & Coconut cluster \\
\hline
\end{tabular}

CgMLST: core genome multilocus sequence typing; CT: cluster type; NA: no information available; ND: none detected; UN: United Nations.

a Representative dataset of the French outbreak.

${ }^{b}$ Excluded from cgMLST with S. Agona scheme.

c Excluded from cgMLST with S.enterica scheme. 
Characteristics of sequenced Bavarian Salmonella Agona isolates and French representative outbreak isolate, Germany, $1993-2018(n=52)$

\begin{tabular}{|c|c|c|c|c|c|c|c|c|}
\hline Sample name & Year & Material & $\begin{array}{l}\text { Isolation } \\
\text { source }\end{array}$ & Country/state & $\begin{array}{l}\text { Country of } \\
\text { origin/travel } \\
\text { history }\end{array}$ & $\begin{array}{l}\text { S. enterica } \\
\text { cgMLST CT }\end{array}$ & $\begin{array}{l}\text { CgMLST } \\
\text { cluster }\end{array}$ & $\begin{array}{c}\text { Epidemiological } \\
\text { link }\end{array}$ \\
\hline SA0053 & 2003 & Aniseed & Tea/raw tea & Germany/Bavaria & NA & 1203 & 1 & Tea outbreak \\
\hline SA0054 & 2003 & $\begin{array}{c}\text { Children } \\
\text { calming tea }\end{array}$ & Tea/raw tea & Germany/Bavaria & NA & 1203 & 1 & Tea outbreak \\
\hline SAoo55 & 2003 & $\begin{array}{c}\text { Children } \\
\text { calming tea }\end{array}$ & Tea/raw tea & Germany/Bavaria & NA & 1203 & 1 & Tea outbreak \\
\hline SA0056 & 2003 & Aniseed & Tea/raw tea & Germany/Bavaria & NA & 1203 & 1 & Tea outbreak \\
\hline SA0057 ${ }^{\mathrm{a}}$ & 2003 & Aniseed organic & Tea/raw tea & Germany/Bavaria & NA & 1203 & $1^{\mathrm{a}}$ & Tea outbreak \\
\hline SA0058 & 2003 & Aniseed organic & Tea/raw tea & Germany/Bavaria & NA & 1203 & 1 & Tea outbreak \\
\hline SA0059 & 2003 & Pectoral tea & Tea/raw tea & Germany/Bavaria & NA & 1203 & 1 & Tea outbreak \\
\hline SA0060 & 2003 & Flatulence tea & Tea/raw tea & Germany/Bavaria & NA & 1203 & 1 & Tea outbreak \\
\hline SAo061 & 2003 & $\begin{array}{l}\text { Pectoral and } \\
\text { cough tea }\end{array}$ & Tea/raw tea & Germany/Bavaria & NA & 1203 & 1 & Tea outbreak \\
\hline SAoo62 & 2003 & $\begin{array}{l}\text { Pectoral and } \\
\text { cough tea }\end{array}$ & Tea/raw tea & Germany/Bavaria & NA & 1203 & 1 & Tea outbreak \\
\hline SA0063 & 2003 & Aniseed & Tea/raw tea & Germany/Bavaria & NA & 1203 & 1 & Tea outbreak \\
\hline SA0064 & 2003 & Aniseed & Tea/raw tea & Germany/Bavaria & NA & 1203 & 1 & Tea outbreak \\
\hline SA0065 & 1993 & Turkey & Food & Germany/Bavaria & NA & 1207 & 7 & NA \\
\hline SAoo66 & 2018 & Stool & Human & Germany/Bavaria & $\begin{array}{l}\text { Thailand, } \\
\text { Cambodia, } \\
\text { Vietnam }\end{array}$ & 1206 & ND & NA \\
\hline
\end{tabular}

CgMLST: core genome multilocus sequence typing; CT: cluster type; NA: no information available; ND: none detected; UN: United Nations. axcluded from cgMLST with S. Agona scheme.

years ago to replace this technique by molecular typing methods such as multilocus sequence typing (MLST), which is able to recognise evolutionary relationships with higher resolution [8]. Furthermore, since many years, pulsed-field gel electrophoresis (PFGE), classifying bacteria based on their universal band pattern after chromosomal restriction, is globally used as a standard molecular technique in outbreak investigations [9-11]. However, despite advantages of molecular techniques, traditional serotyping is still universally used and provides an important historical context.

Beyond PFGE and MLST, variable number of tandem repeats (VNTRs) proved to be suitable molecular targets for assessing genetic polymorphisms within bacterial species $[12,13]$. The multilocus variable number of tandem repeats (MLVA) technique, as a form of VNTR typing showed increased analysis depth in outbreak investigations and proved to be suitable for important Salmonella serovars such as Enteritidis, Typhimurium or Dublin [14-16]. However, the variability of protocols and targets hindered comprehensive standardisation, although efforts towards this are ongoing $[17,18]$. All these techniques provide reliable first level classification and are discriminative enough to investigate epidemiologically well-defined outbreaks.

Nonetheless, in the meantime, a number of studies have shown that whole genome sequencing (WGS) gives the highest resolution for outbreak investigation, especially if case distribution is diffuse with respect to geographical area or time frame of occurrence $[7,19,20]$. For implementation of WGS in S. Agona outbreak investigations and molecular surveillance, it has to be taken into account that this serovar is monophyletic, more homogeneous, as well as evolutionarily younger than most other well-investigated pathogenic serovars $[21,22]$.

In 2017 , three feed samples (rapeseed meal) of a factory in the district of Lower Bavaria were submitted to the Bavarian Health and Food Safety Authority. Culture-based species and serovar identification detected $S$. Agona in all three samples. In December 2017, an outbreak of the same serovar was reported in France, attributable to 37 French cases and two international cases, caused by infant milk products of a French supplier and traceable to one single French production facility $[23,24]$.

The aim of the current WGS investigation, using next generation sequencing (NGS) was to verify potential clonality of the Bavarian isolates, to exclude any connection between the Bavarian feed samples and the simultaneous French outbreak and to gain a more precise insight into the genetic diversity of $S$. Agona collected in Bavaria over the past 25 years. 


\section{FIGURE 1}

Minimum spanning tree of the core genome multilocus sequence type allelic profiles of Salmonella Agona strains, including 51 Bavarian isolates, a representative isolate of an infant-milk-caused outbreak in France and the reference strain SL-483, Germany, 1993-2018 ( $\mathrm{n}=53$ isolates)

Animal feed
Cattle
$\bigcirc$ Chicken
Food
$\bigcirc$ Human
Nutritional supplement
Reference
$\bigcirc$ Spices
Tea/raw tea
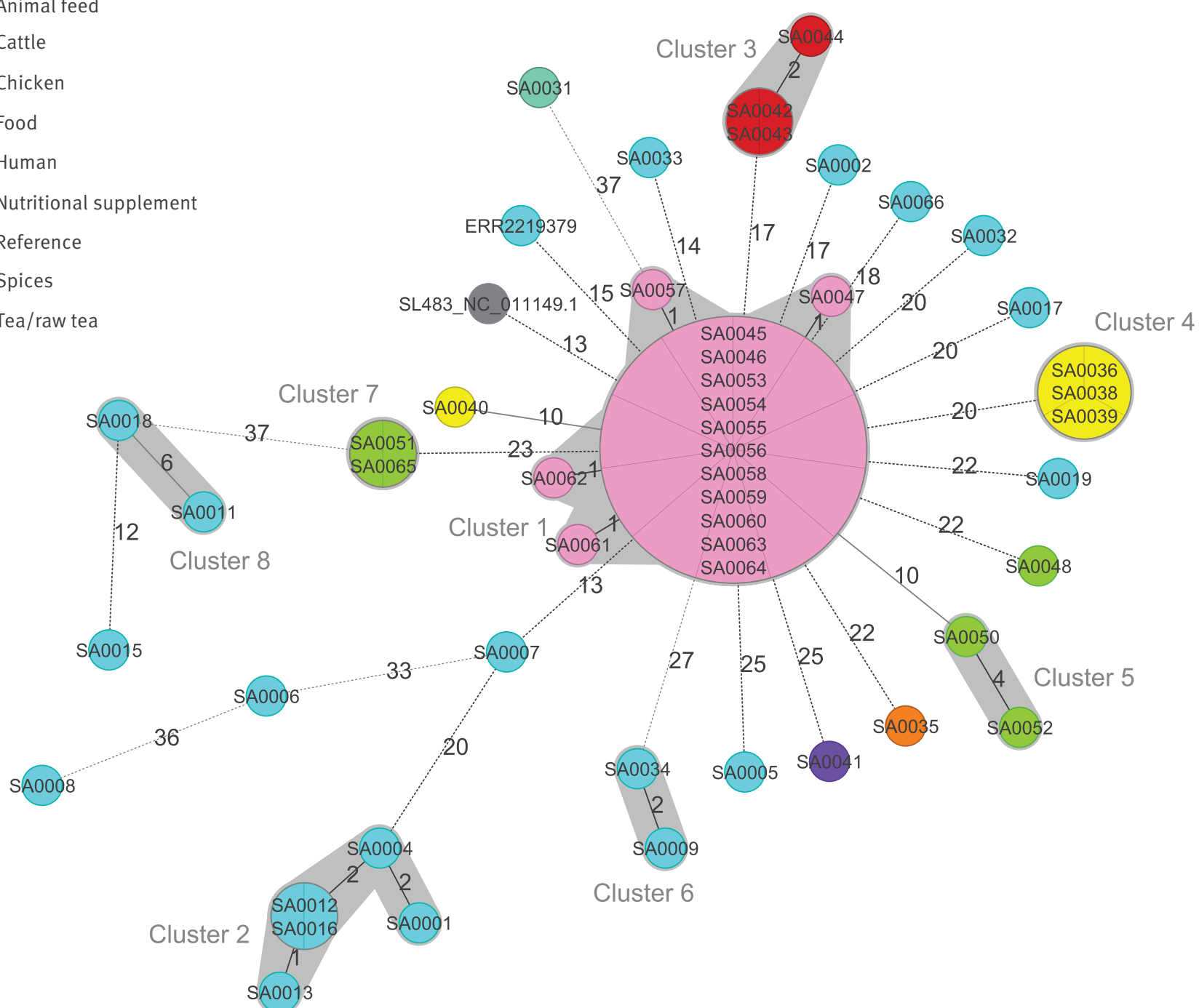

CgMLST: core genome multilocus sequence typing.

Bavarian S. Agona samples have the prefix SA. The representative isolate of the infant-milk outbreak in France [23] is ERR2219379. The National Center for Biotechnology Information (NCBI) reference genome of strain SL-483 has GenBank accession number NC_011149.1.

The analysis and tree were obtained with the public Ridom-SeqSphere+-integrated S. enterica cgMLST scheme of 3,002 target loci.

On the tree, allele distances between samples are indicated. Clusters of samples with maximum seven alleles distance are shaded in grey. Samples are colour coded by their isolation source, as given in the legend. 


\section{Methods}

Isolates and sequence data used in the analyses For this observational retrospective study, we used 48 S. Agona isolates dated from 1993 to 2018 from our strain collections to investigate the three isolates of the Bavarian rapeseed meal from 2017 in a wider context. The total 51 isolates were all the $S$. Agona isolates available for us. As many diagnostic laboratories exist in Bavaria, our isolates were not representative for the occurrence of $S$. Agona in this federal state, where, for example, 99 cases of human infections had been officially notified in the 2011 to 2018 period alone.

The 48 isolates that we employed were either from humans, food, feed, animals or the environment. A total of 28 thereof were outbreak-related. Outbreak isolates belonged to four epidemiologically-linked events represented by 15 , three, three and two isolates as well as an additional five isolates with a suspected epidemiological connection.

The 48 isolates from 1993 to 2018 were studied by WGS together with the three 2017 isolates of the Bavarian rapeseed feed. Raw NGS data of the published representative isolate of the French outbreak [24], available under European Nucleotide Archive (ENA) accession ERR2219379, were also added to the bioinformatics analyses.

For the evaluation of necessary analysis depth, 70 NGS raw datasets from various European outbreaks, published by Zhou et al. 2013 [21], available under National Center for Biotechnology Information (NCBI) bioproject PRJEB1944, were added to the data analysis as well.

To distinguish the subset of isolates derived from our strain collections, or data thereof, from the data published by Zhou et al. [21], we further refer to our $51 \mathrm{~S}$. Agona isolates/strains as 'Bavarian'.

\section{Species and serovar identification}

All Bavarian S. Agona strains were cultured on Columbia sheep blood agar (Oxoid, Wesel, Germany) and identified by matrix-assisted laser desorption/ionisation time-of-flight mass spectroscopy (MALDI-TOF MS; Bruker, Bremen, Germany). Somatic (O) and flagel$\operatorname{lar}(\mathrm{H})$ antigens were identified by using slide agglutination (antisera provided by Sifin, Berlin, Germany) according to the White-Kauffmann-Le Minor scheme [25].

\section{Next generation sequencing}

Salmonella Agona isolates were freshly grown on blood agar plates. One inoculation loop of bacterial material was suspended in $50 \mu \mathrm{L}$ phosphate buffered saline (PBS) and cells were pre-treated with $1 \mu \mathrm{g}$ lysozyme for $15 \mathrm{~min}$ at $37^{\circ} \mathrm{C}$ followed by a 2 hour incubation step at $65^{\circ} \mathrm{C}$ with $200 \mu \mathrm{L}$ incorporation buffer, $200 \mu \mathrm{L}$ lysis buffer, $30 \mu \mathrm{L} 20 \mathrm{mg} / \mathrm{mL}$ Proteinase $\mathrm{K}$ and $10 \mu \mathrm{L} 10 \mathrm{mg} /$ $\mathrm{mL}$ ribonuclease (RNase) A (all reagents from Promega,
Mannheim, Germany). Genomic DNA (gDNA) was then isolated with the Maxwell 16 LEV Blood DNA Kit on the Maxwell 16 instrument (Promega, Mannheim, Germany) according to manufacturer's instructions with Tris buffer for gDNA elution.

Whole genome libraries for NGS were prepared using the Nextera XT kit (Illumina, San Diego, California, US). Next generation sequencing was performed on the Illumina MiSeq with $2 \times 250 \mathrm{bp}$ paired-end reads. Sequencing runs were evaluated for quality using the Illumina SAV Software.

Sequencing data were uploaded to the NCBI sequence read archive (SRA) [26], under BioProject PRJNA473689.

\section{Multilocus sequence typing analyses}

Core genome MLST (cgMLST) of reads was performed with Ridom SeqSphere+Software (Ridom, Munster, Germany [27]) with default settings for trimming and velvet assembly. For the assignment of cgMLST alleles, two different schemes were used: (i) a publicly-available S. enterica (species level) cgMLST scheme designed by Enterobase and (ii) an in-house serovar-specific cgMLST scheme.

Enterobase-designed Salmonella enterica core genome multilocus typing scheme

The publicly available species-specific SeqSphere + software-implemented $S$. enterica cgMLST scheme with the 3,002 target loci developed as Salmonella cgMLST v2 scheme by Enterobase was employed to analyse sequencing data $[28,29]$.

In-house serovar-specific Salmonella Agona core genome multilocus typing scheme

An in-house developed serovar-specific S. Agona scheme, based on reference genome NC_011149.1 of strain SL-483 and query genomes NC_022991.1, NZ_ CP015024.1, NZ_CP011259.1, was generated using the following default filter thresholds.

For the reference genome filter thresholds: (i) minimum length: 60 bases; (ii) start codon and single stop codon required at beginning and end of gene; (iii) homologous/paralogous gene filter, excluding multiple copies of a gene with basic local alignment search tool (BLAST) overlap $\geq 100$ bp or identity $\geq 90 \%$; (iv) overlap filter, excluding overlap with other genes $>4$ bases.

For the query genome filters thresholds: (i) start and stop codon required at beginning and end of gene; (ii) BLAST hit locus overlap $=100 \%$ and identity $\geq 90 \%$ in every query genome; (iii) BLAST options: word size $=11$, mismatch penalty $=-1$, match reward $=1$, gap open costs $=5$, gap extension costs $=2$.

Thereby, the final scheme resulted in 4,111 target loci. New alleles and sequence types (ST) were submitted to the nomenclature server for the public scheme. 


\section{FIGURE 2}

Maximum likelihood tree resulting from the whole genome single nucleotide polymorphism-based phylogenetic analysis of Salmonella Agona strains including 51 Bavarian isolates, a representative isolate of an infant milk-caused outbreak in France and the reference strain SL-483, Germany, 1993-2018 ( $\mathrm{n}=53$ isolates)

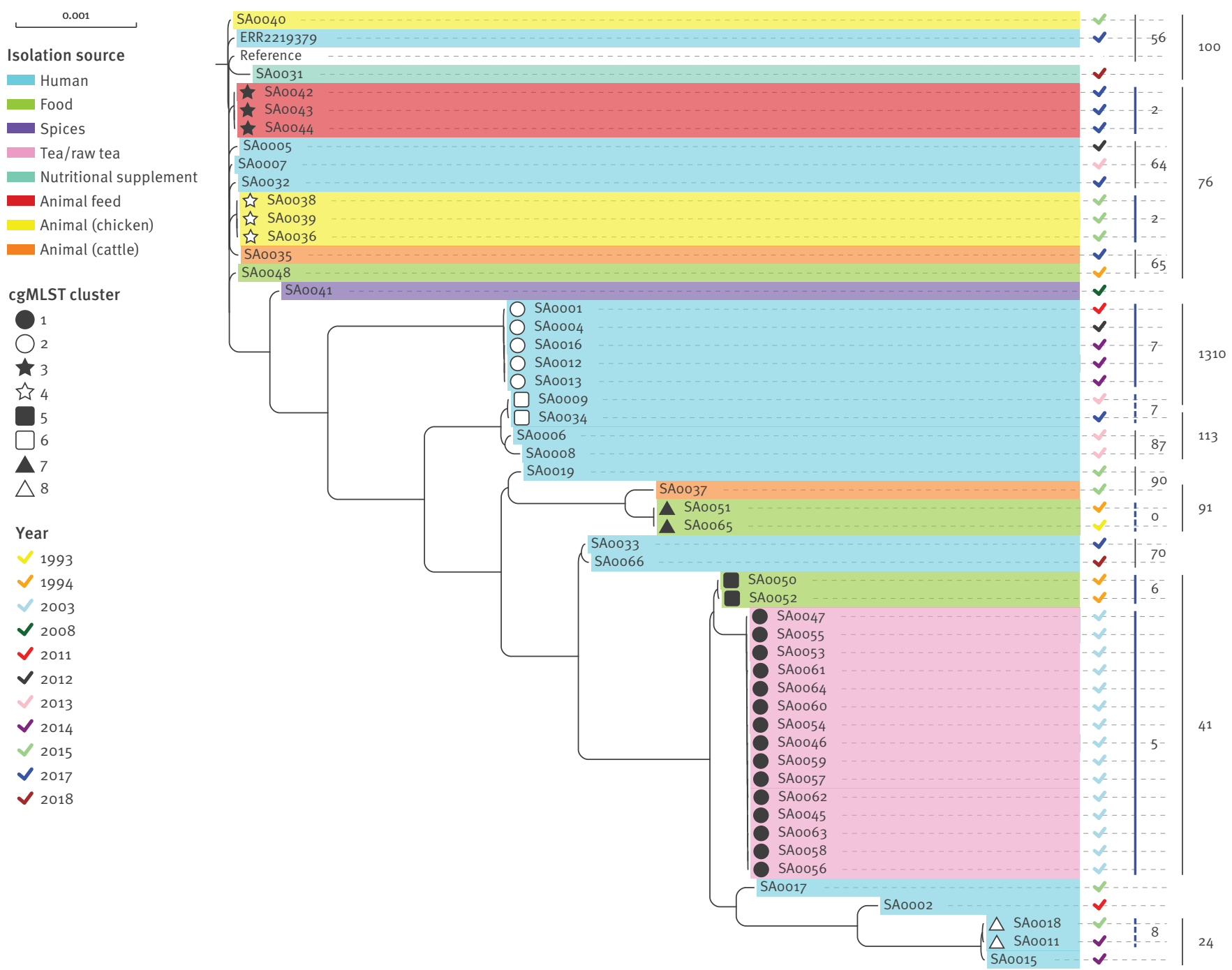

cgMLST: core genome multilocus sequence typing.

Bavarian S. Agona samples have the prefix SA. The representative isolate of the infant milk outbreak in France [23] is ERR2219379. The National Center for Biotechnology Information (NCBI) reference genome of strain SL-483 has GenBank accession number NC_011149.1 and figures in the tree as 'reference'.

In the tree, samples are colour coded according to isolation source, S. Agona cgMLST cluster and collection year as given in the legend.

The general scale bar indicates 0.001 substitutions per site (597 SNPs), based on an alignment of 596,849 positions.

SNP distance bars between specific samples are indicated on the right side. Blue vertical SNP scale bars indicate epidemiologically-linked cgMLST clusters, blue vertical dashed lines indicate cgMLST clusters without epidemiological link and black verticallines indicate exemplary distances between non clustered samples. 
Assessment of relationships between isolates' core genome multilocus sequence type allelic profiles by minimum spanning trees

cgMLST typing results were visualised in minimum spanning trees (MSTs), excluding all samples in the respective scheme, not fulfilling 'good target' quality control (QC) for>90\% for the scheme's target loci (=90\% of targets present in the isolate, same length as reference $+/-3$ triplets, without ambiguities and without frame shifts in consensus, Table 1). A cluster was defined as a group of closely related cgMLST-analysed isolates in both schemes with a single-linkage threshold of $\leq 7$ alleles. This was the default distance threshold for the software-implemented public S.enterica scheme and was adopted for direct comparison for the $S$. Agona scheme. During typing with the $S$. entericacgMLST scheme, the SeqSphere + software assigns an existing cluster type (CT) to each isolate with an allelic distance $\leq 7$ to an already established CT founder profile on the central nomenclature server. Otherwise, a new CT is established, uploaded and the isolate becomes the founder of this CT $[30,31]$.

Multilocus sequence typing

In silico MLST analysis of NGS data was performed with the standard seven-gene target scheme [32].

\section{Single nucleotide polymorphism phylogeny}

Whole genome (wg) single nucleotide polymorphism (SNP)-based phylogeny was calculated using the run_snp_pipeline script of the PHEnix pipeline by Public Health England [33]. It includes trimming with trimmomatic [34], mapping to the reference genome NC_011149.1 of strain SL-483 with bwa-mem mapping [35] with default settings, variant calling and filtering (frequency $\geq 0.9$, mapping quality score $\geq 30$, read depth $\geq 10$ ) by Genome Analysis Toolkit (GATK)2 Unified Genotyper [36]. Variant calls for all SNP positions passing filters and all positions not passing filters were extracted and SNPs were concatenated to alignments with the vcf2fasta-script from the same pipeline, allowing $\leq 90 \%$ missing data per sample and $\leq 20 \%$ missing data per position throughout all samples. Maximum likelihood (ML) trees were generated from SNP alignments by RaxML [37], including 100 bootstrap replicates.

\section{Results}

Next generation sequencing of the 51 Bavarian S. Agona isolates (named with prefix SA), collected between 1993 and 2018 revealed high quality reads and reference genome coverage of 28-171 fold. In our bioinformatics data analyses, we included the published NGS raw data (ENA accession: ERR2219379) of an isolate from a case of the infant-milk-caused outbreak originating from a France-based manufacturer [24]. This isolate served as a representative of the French outbreak. All isolates were typed by in silico MLST, resulting in ST13, the typical ST for serovar Agona [8].
In a next step, the isolates were typed with Ridom SeqSphere+software with the public $S$. enterica cgMLST scheme, consisting of Enterobasedeveloped 3,002 target loci (Figure 1). Additionally, wg SNP analysis was performed to investigate the phylogenetic relationship in highest possible resolution (Figure 2).

The MST from results of the $S$. enterica cgMLST scheme, including all samples exceeding the target-QC cut-off, revealed in total eight clusters with maximum six alleles difference (Figure 1). The four most relevant clusters (1-4) comprised three to 15 samples with a maximum within cluster difference of zero to five alleles (Table 2). Each of the remaining four clusters $(5-8)$ included two samples and internal distances ranging from zero (cluster 7) to six alleles (cluster 8).

None of the 51 Bavarian isolates collected from 19932018 clustered with the representative sample from the recent outbreak in France. The French representative sample ERR2219379 differed from the Bavarian samples in at least 15 alleles/40 SNPs. This difference was observed to distinguish unrelated isolates and clusters from different years or with different epidemiological origins throughout the whole sample set (Figure 1, Figure 2, Table 1).

The three Bavarian feed isolates collected in 2017 from rapeseed cake (SA0042, SA0043, SAo044) built up a distinct cluster with two alleles maximum distance (Figure 1, cluster 3), corresponding to two SNPs in the wg SNP analysis (Figure 2). They differed from the nearest neighbours outside the clusters in at least 17 alleles/37 SNPs (Table 2).

Furthermore, some of the Bavarian strains, isolated in former years, aggregated in epidemiologically described clusters, although the corresponding isolates available in our strain collection for sequencing mainly covered food or veterinary samples and corresponding human isolates were not available for analysis. Most eye-catching, the isolates of the biggest cluster with 15 samples (Figure 1, cluster 1) were all isolated from tea or raw tea products, connected to a diffuse outbreak caused by aniseed-fennel-caraway tea products in 2002-03. The outbreak investigation at that time identified contaminated raw tea imported from Turkey as source [2,3]. All isolates of this cluster were closely connected with zero to one allele singlelinkage distance (Figure 1) and three alleles or five SNPS (Figure 2) maximum distance within the cluster. They showed at least 10 alleles/26 SNPs to all other non-connected isolates (Table 2 ).

The five isolates of cluster 2 with single-linkage distances of zero to two alleles and maximum intra-cluster distance of five alleles or seven SNPs were obtained from asylum seekers between 2011 and 2015 (Table 1). Details of their travel history regarding countries or period are unknown. Hence the slightly higher 
Allele and single nucleotide polymorphism differences within and between clusters of the sequenced

Bavarian Salmonella Agona isolates, Germany, 1993-2018 ( $\mathrm{n}=34$ isolates)

\begin{tabular}{|c|c|c|c|c|c|c|c|c|c|}
\hline \multirow[b]{2}{*}{ Measure } & \multirow[b]{2}{*}{ Method } & \multicolumn{8}{|c|}{$\begin{array}{l}\text { Cluster ID } \\
\text { (number of isolates) } \\
\text { Epidemiological link }\end{array}$} \\
\hline & & $\begin{array}{l}\text { Cluster } 1 \\
\qquad \begin{array}{l}\text { (15) } \\
\text { Tea } \\
\text { outbreak }\end{array}\end{array}$ & $\begin{array}{c}\text { Cluster } 2 \\
\text { (5) } \\
\text { Travel } \\
\text { cluster }\end{array}$ & $\begin{array}{l}\text { Cluster } 3 \\
\text { (3) } \\
\text { Feed } \\
\text { cluster }\end{array}$ & $\begin{array}{l}\text { Cluster } 4 \\
\text { (3) } \\
\text { Chicken } \\
\text { cluster }\end{array}$ & $\begin{array}{l}\text { Cluster } 5 \\
\text { (2) } \\
\text { Coconut } \\
\text { cluster }\end{array}$ & $\begin{array}{c}\text { Cluster } 6 \\
\text { (2) } \\
\text { None } \\
\text { detected }\end{array}$ & $\begin{array}{c}\text { Cluster } 7 \\
\text { (2) } \\
\text { None } \\
\text { detected }\end{array}$ & $\begin{array}{c}\text { Cluster } 8^{a} \\
(2)^{a} \\
\text { None } \\
\text { detected }\end{array}$ \\
\hline \multirow{3}{*}{$\begin{array}{l}\text { Within cluster } \\
\text { distance: median } \\
\text { (min-max) }\end{array}$} & $\begin{array}{c}\text { Salmonella enterica } \\
\text { CgMLST }\end{array}$ & $0(0-3)$ & $2(0-5)$ & $0(0-2)$ & $0(0-0)$ & $4(4-4)$ & $2(2-2)$ & $0(0-0)$ & $6(6-6)$ \\
\hline & S. Agona cgMLST & $0(0-3)$ & $2(0-6)$ & $1(0-3)$ & $0(0-2)$ & $3(3-3)$ & $2.5(0-5)$ & $0(0-0)$ & None $^{a}$ \\
\hline & SNP phylogeny & $1(0-5)$ & $3.5(0-7)$ & $2(0-2)$ & $2(0-2)$ & $6(6-6)$ & $7(7-7)$ & $0(0-0)$ & $8(8-8)$ \\
\hline \multirow{3}{*}{$\begin{array}{l}\text { Min distance to } \\
\text { nearest neighbour }\end{array}$} & S. enterica cgMLST & 10 & 20 & 17 & 20 & 10 & 27 & 23 & 12 \\
\hline & S. Agona cgMLST & 15 & 30 & 25 & 28 & 15 & 33 & 37 & None $^{a}$ \\
\hline & SNP phylogeny & 21 & 47 & 37 & 37 & 21 & 49 & 59 & 22 \\
\hline \multirow{3}{*}{$\begin{array}{l}\text { Nearest neighbour } \\
\text { outside cluster in } \\
\text { question }\end{array}$} & S. enterica cgMLST & $\begin{array}{l}\text { SA0040, } \\
\text { SA0050 } \\
\quad \text { (in } \\
\text { cluster 5) }\end{array}$ & SA0007 & $\begin{array}{l}\text { SAo0 53, } \\
\text { SAo054, } \\
\text { SAo0 55, } \\
\text { SA0058 } \\
\quad \text { (all } \\
\text { cluster 1) }\end{array}$ & $\begin{array}{l}\text { SA0055 } \\
\text { (in } \\
\text { cluster } \\
\text { 1) }\end{array}$ & $\begin{array}{l}\text { SAoo 053, } \\
\text { SAo0 054, } \\
\text { SAo 055, } \\
\text { SA0058 } \\
\text { (all } \\
\text { cluster 1) }\end{array}$ & $\begin{array}{l}\text { SA0053 } \\
\quad \text { (in } \\
\text { cluster1) }\end{array}$ & $\begin{array}{l}\text { SA0055, } \\
\text { SA0056 } \\
\text { (all } \\
\text { cluster1) }\end{array}$ & SA0015 \\
\hline & S. Agona cgMLST & $\begin{array}{l}\text { SA0007, } \\
\text { SA0050 } \\
\text { (in } \\
\text { cluster 5) }\end{array}$ & $\begin{array}{c}\text { SA0047 } \\
\text { (in cluster } \\
1 \text { ) }\end{array}$ & $\begin{array}{c}\text { SAo047 } \\
\text { and } \\
\text { SAo058 } \\
\text { (both in } \\
\text { cluster 1), } \\
\text { SA0007 }\end{array}$ & SA0007 & $\begin{array}{l}\text { SAo055, } \\
\text { SA0056 } \\
\text { (all } \\
\text { cluster 1) }\end{array}$ & $\begin{array}{l}\text { SA0053 } \\
\text { (in } \\
\text { cluster 1) }\end{array}$ & SA0007 & None $^{a}$ \\
\hline & SNP phylogeny & $\begin{array}{l}\text { SA0050 } \\
\text { (in } \\
\text { cluster 5) }\end{array}$ & $\begin{array}{l}\text { SAoo47 } \\
\text { (in } \\
\text { cluster 1) }\end{array}$ & SA0007 & SA0007 & $\begin{array}{l}\text { SA0057 } \\
\text { (in } \\
\text { cluster 1) }\end{array}$ & $\begin{array}{l}\text { SA0047 } \\
\text { (in } \\
\text { cluster 1) }\end{array}$ & $\begin{array}{l}\text { SA0018 } \\
\text { (in } \\
\text { cluster 8) }\end{array}$ & SA0015 \\
\hline
\end{tabular}

CgMLST: core genome multilocus sequence typing; max: maximum; min: minimum; SNP: single nucleotide polymorphism.

a Cluster 8 not detected in S. Agona cgMLST due to exclusion of sample SA0018.

variation of their allelic/SNP distances than in other point-sourced epidemiologically linked clusters is not surprising. Cluster 4 consists of three samples with zero alleles/SNPs difference to each other but at least 20 alleles/45 SNPs difference to the other samples. Isolates in this cluster shared a clear epidemiological link, as samples originated from chicken faecal samples, collected in 2015 in different laying hen flocks of one Bavarian egg producer.

Clusters 5, 6, 7 and 8 only consist of two isolates each, respectively. Cluster 5 was built up from two shredded coconut samples from 1994 with four alleles/six SNPs difference which likely have an epidemiological link although information on their origin or supplier is not available. Isolates from two human patients without epidemiological link clustered together with two alleles (cluster 6) and seven SNPs distance. Cluster 7 came from two genetically identical strains from food samples from 1993-94 (shredded coconut and turkey) for which no epidemiological link is known. One additional pair of closely related human samples (SA0011 and SA0018) with reported travel history to Eritrea (SA0018) and an unspecified country (SAoo11) built cluster 8 with six alleles and eight SNPs difference.

To evaluate the needed resolution of the cgMLST analysis regarding analysed genomic content and consequential cluster demarcation, the species level typing with the public $S$. enterica cgMLST scheme, consisting of Enterobase-developed 3,002 target loci was compared with an in-house generated ad hoc serovar Agona-specific cgMLST scheme with 4,111 target loci (Figure 3). Typing with both schemes was performed using the SeqSphere+software algorithms. As no empiric threshold was defined prior the analysis for the serovar-specific scheme it was used with the same thresholds as the species-specific scheme. For the comparison of the data analysis approaches, 70 published NGS raw datasets of isolates described by Zhou 
et al. [21] were added in both approaches to widen the view on cluster definition and concordance by genomic and epidemiological data on a supra-regional level. This dataset covered S. Agona isolates from 1952 to 2010 covering several European outbreaks. The results of the public S. enterica cgMLST scheme (Figure $3 \mathrm{~A}$ ) are very similar to those of the in-house $S$. Agona cgMLST scheme (Figure 3B) and SNP-profiling (Figure 2). All Bavarian and published outbreak clusters were detected by both schemes (Figure 3 A and B). Therefore, the usage of the public $S$. enterica scheme was generally evaluated as practical for $S$. Agona outbreak investigations.

As SA0018 was excluded from the serovar-specific cgMLST due to shortfall below the $90 \%$ good target threshold for the $S$. Agona scheme target loci, cluster 8 detected in species-specific cgMLST (Figure 1, Figure $3 \mathrm{~A})$ was not apparent in serovar-specific cgMLST (Figure 3B).

Due to a smaller number of target loci in the public cgMLST scheme, allele distances between samples were generally lower than with the serovar-specific scheme. Two epidemiologically unrelated clusters linked by an unrelated Irish environmental isolate (ERS180349) (Figure 3B, clusters 2 and 12) could not be delimitated clearly in the $S$. entericascheme with a default cluster threshold of seven alleles (Figure $3 \mathrm{~A}$, cluster $2+12)$. Those two clusters - one consisting of Bavarian patient isolates with travel history to African or Arabian countries described above (cluster 2; Table 1 and 2) and one described by Zhou et al. (Figure 3B, cluster 12 ), originating from a large multicountry 2008-09 outbreak originating from Ireland [21] - could more clearly be separated with the in-house cgMLST S. Agona scheme (Figure 3B, clusters 2 and 12), using the same cluster threshold. In the specieslevel cgMLST scheme, the software assigns CTs for all samples. These were different for most isolates of the two unrelated clusters, suggesting two different sources for these two clusters, additional to the epidemiological information.

\section{Discussion}

Our high resolution WGS-based analysis approach by either wg SNP-profiling or species- or serovar-specific cgMLST delivered good and reliable results for typing $S$. Agona isolates and evaluating their affiliation to, or delineation from, outbreak clusters. We could thereby exclude a connection between the Bavarian feed sample isolates from 2017 and a large outbreak due to infant milk products, which occurred at the same time in France and other European countries. Furthermore, we could confirm S. Agona epidemiological clusters from former years as well as identify previously unrecognised clusters. As recently shown for serovar S. Enteritidis [38], cgMLST was a standardisable and easily applicable to $S$. Agona and could reach an analysis depth comparable to wg SNP profiling.
Recently, cgMLSTwas published as a tool with reasonable resolution for investigations of Salmonella population structure [29]. Thereby, the extensively curated $S$. enterica based scheme was made available on a publicly accessible website [28]. The same scheme was implemented in the Ridom SeqSphere+Software too, although with own allele calling and clustering rules and was used in this study to compare the resolution of species- and serovar-specific typing with our inhouse developed serovar-specific scheme. It could be shown that even on species level all outbreaks were clearly identified by both schemes. Only two independent outbreak clusters could not be delimitated clearly anymore by the publicly available scheme, due to the lower number of species-specific target loci and the used cluster threshold.

Of course, more focused typing schemes or approaches like serovar-level cgMLST or wg SNP genotyping deliver more detailed typing results catching at least the portion of the serovar-specific diversity manifested in the core- or reference-genome. This is particularly true for monomorphic organisms such as S. Agona, for which mobile elements that are mostly not covered by core genome or single reference-based approaches generate a lot of diversity [21], or for situations when single samples are to be inferred as part of specific clusters or not, especially when epidemiological information is not fully discriminative. Consequently, the serovar-specific in-house scheme developed for this investigation, worked very well for the analysed sample set. However, due to the lack of target curation and its creation from only one reference and three query genomes, it will need further optimisation and testing with diverse sample sets for suitability on a broader scale. This can be assumed from the fact that four isolates had to be excluded from typing due to shortfall below the $90 \%$ good target threshold using the in-house scheme, but only one isolate using the publicly available scheme (Table 1). The use of a publicly available scheme whose target loci underwent extensive curation, like the Enterobase S. enterica scheme [29], can render suitable and reliable results even in cases where serotyping has not yet been performed or in the event of the lack of a specific reference.

Generally, cluster definition is based on a single-linkage allelic difference threshold. The software implements a proposed default threshold of seven alleles for the $S$. enterica scheme. This was adopted for the $S$. Agona scheme for comparison and as no empirically tested threshold was established for this scheme before. However, as shown with the high-resolution in-house S. Agona cgMLST scheme, clusters with a clear epidemiological link did not show more than four alleles and six SNPs difference. Therefore, a cluster with a distance above four, but below the threshold of seven alleles (e.g. Figure 3B, clusters 6 and 11), may contain falsely grouped samples. Thus, the cluster thresholds in both schemes could be decreased down from seven based on the analysed sample set. 


\section{FIGURE 3}

Minimum spanning trees obtained with different typing schemes (Panels A and B) of the core genome multilocus sequence type allelic profiles of 51 Bavarian Salmonella Agona isolates, a representative isolate of an infant milk outbreak in France, the reference strain SL-483 and 70 isolates from various European outbreaks $(n=123$ isolates)

\section{A. Result from the Ridom-SeqSphere + -integrated S. enterica cgMLST scheme of 3,002 target loci}

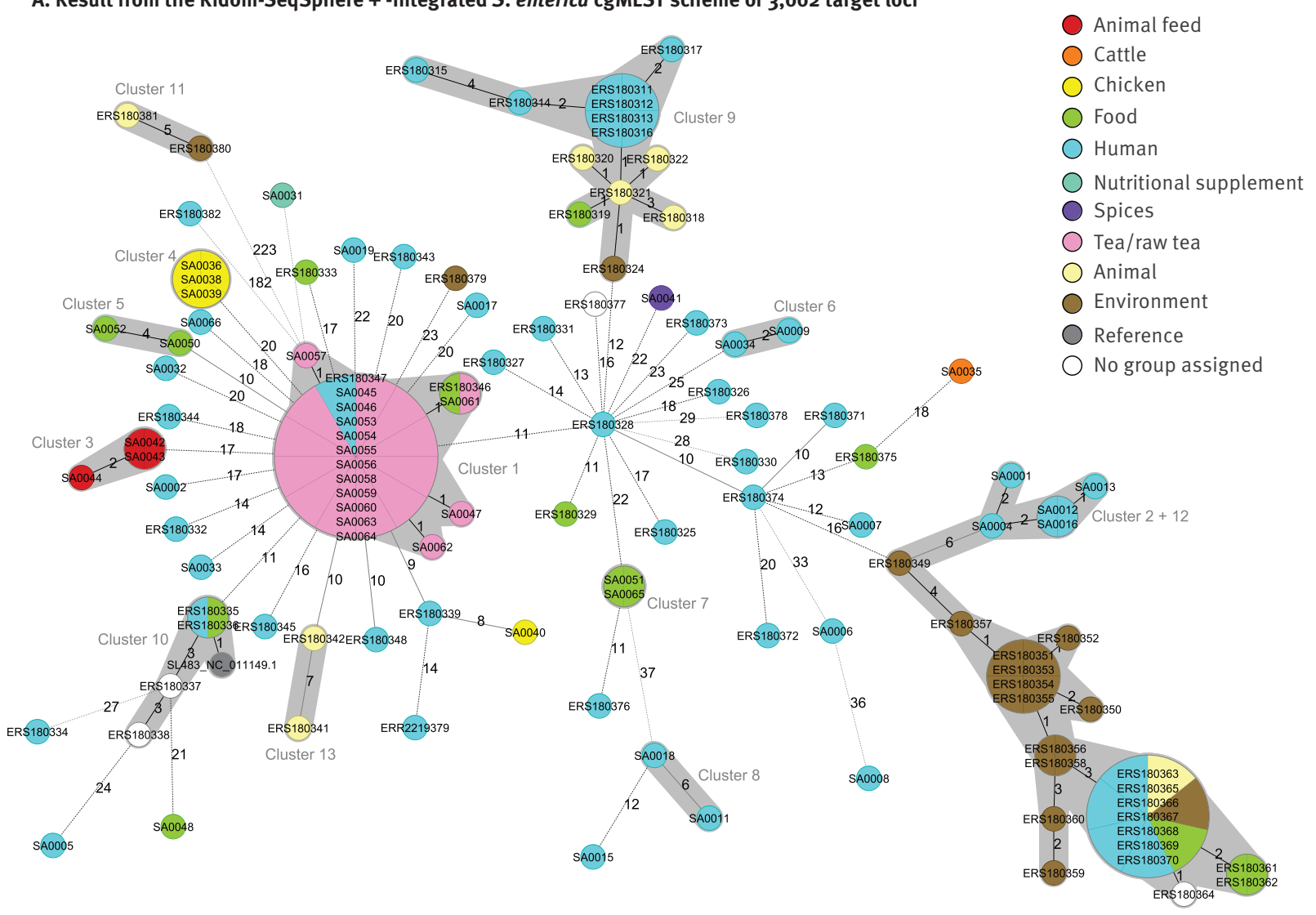

B. Result from the in-house $S$. Agona serovar-specific cgMLST scheme of 4,111 target loci

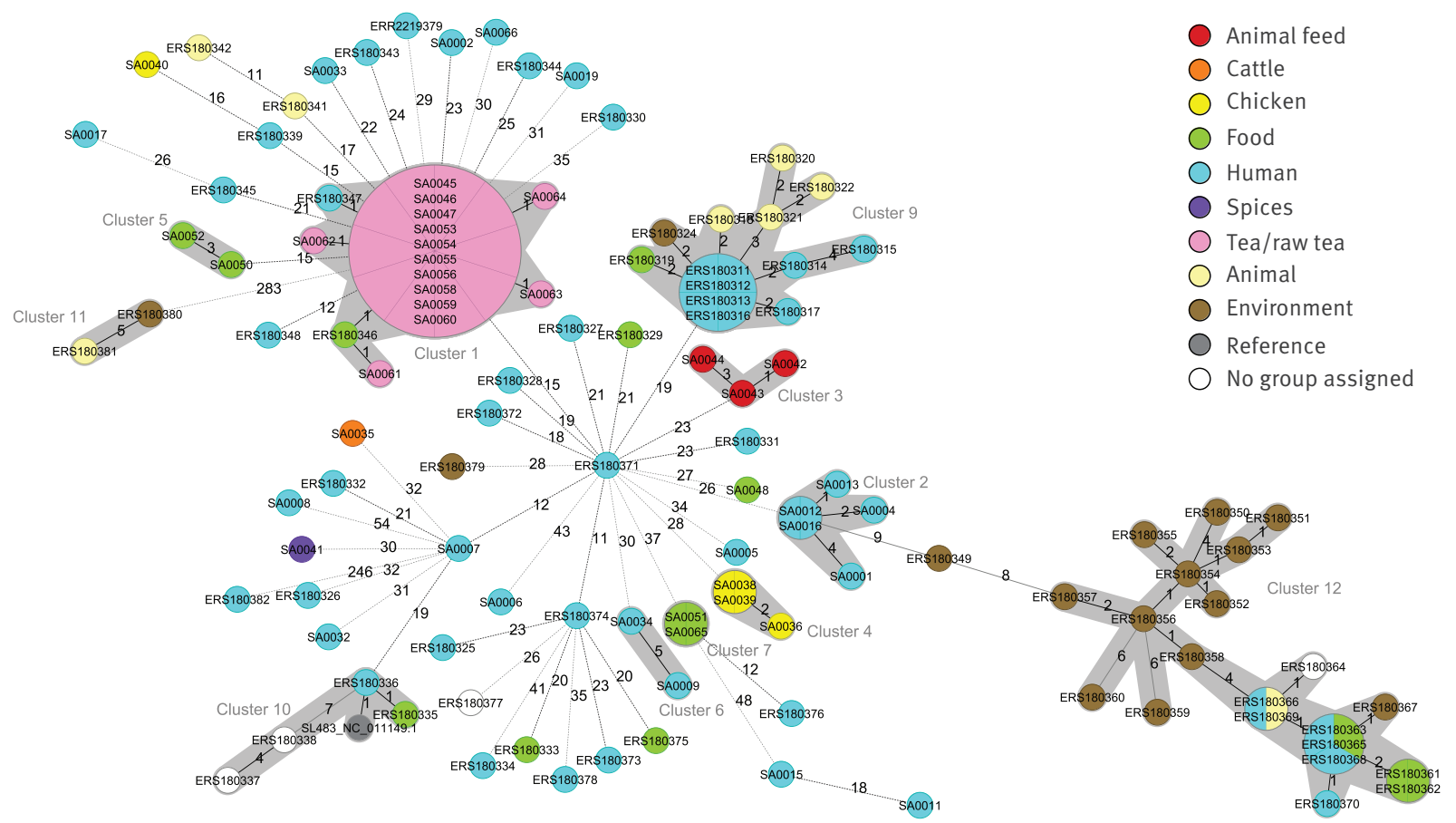

CgMLST: core genome multilocus sequence typing.

a Due to shortfall below the $90 \%$ good target threshold for the S. Agona scheme target loci, SAoo18 does not figure in panel B, which shows a total of 122 isolates.

Bavarian S. Agona samples have the prefix SA. The representative isolate of the infant milk outbreak in France [23] is ERR2219379. The National Center for Biotechnology Information (NCBI) reference genome of strain SL-483 has GenBank accession number NC_011149.1. The 70 isolates from various European outbreaks are published in a separate study [21].

Allele distances between samples are indicated and clusters of samples with minimum spanning distances of zero to seven alleles are shaded in grey. Samples are colour coded by their isolation source, as given in the legend. 
However, theoretically a cluster threshold should be lower in a scheme with less loci like the S. enterica scheme (3,002 loci) than in a scheme with more loci like the $S$. Agonas scheme (4,111 loci). A decreased cluster threshold would be in accordance with the special genetic characteristics of $S$. Agona, which emerged more recently and is more monomorphic than most other serovars [21]. However, while facilitating cluster delimitation in S. Agona, a decreased threshold would, especially in the $S$. enterica scheme, very likely impair clustering in the case of more heterogeneous serovars or when linked isolates have evolved over a longer time period.

Cluster types assigned by the publicly available $S$. enterica scheme are intended to roughly classify genetically similar samples in an easy way. This is very helpful and an important step towards standardisation, also in terms of inter-laboratory communication and for alerts concerning the detection of a known CT. In the case of the two epidemiologically unrelated clusters 2 and 12 (Figure 3B), which were not clearly delimited by the public $S$. entericascheme (Figure $3 A$, Cluster $2+12$ ), the software-assigned CT values for each isolate were mainly distinct for the two clusters (cluster 2: CT-1195, cluster 12: (T-25). Hence, using the CT as a simplification measure to distinguish between clusters from different sources helped, although the clusters were difficult to resolve at least with a MST and the applied threshold for single-linkage clustering. However, due to the complexity of NGS data the logical principle on which such a simplification, like using the CT value, is based, always has to be considered for its correct use, as this can also result in false inclusion into or exclusion from a cluster. Indeed, the CTs are assigned depending on an isolate's proximity in terms of allelic distance to a specific CT founder allele profile or by incremental expansion of the nomenclature when a new isolate exhibits a not yet known and sufficiently different allele combination $[30,31]$. The incremental nomenclature expansion together with the fact that only the distance to the nearest CT founder is reflected in the CT assignment of new isolates can lead to the assignment of the same CT to isolates with allelic distances above the threshold or of different CTs to isolates below a cluster threshold. This may be for example the case for isolate ERS180350, correctly grouped within the Irish outbreak cluster of 2008 with common CT-25 (Figure 3B, cluster 12), but being tagged with a deviant CT (CT-1224). Furthermore, microbial evolution can be different in certain epidemiological niches which cannot fully be covered by such simplification.

Due to these limitations, outbreak investigations should generally avoid relying solely on simplifications like CT values, but also include visual inspection of cluster formation in trees to avoid overlooking of connected samples. Classifications by clusters or simplified measures such as CTs should always be interpreted with caution, especially when allelic or SNP distances near an empirically tested cluster threshold occur. Moreover, particularly, but not only, for organisms with specific genetic characteristics such as $S$. Agona, empirical as well as epidemiological data should always be taken into account in addition to the molecular data [39].

Our analysis also shows that if a common reference or close genetic relationship for a specific set of isolates is known, high resolution approaches facilitate analysis and enable clear grouping of individual isolates in dispute, whereas the versatility of genetically broader approaches enables more standardised results for more heterogeneous sets of isolates. To reduce interpretation complexity and further extend fast and easy usability of NGS approaches for public health analyses, further standardisation and harmonisation between laboratories are nevertheless required. Many researchers and authorities have already realised this, but the implementation and ongoing optimisation will be a huge effort in the forthcoming years.

Concluding, consistent with results obtained for other serovars and species, wg SNP profiling as well as serovar- or species-specific cgMLST can be used for reasonable, reproducible and reliable high resolution classification of S.Agona WGS data to detect outbreak clusters. We showed this with a representative dataset from regional and international sources covering human, food, feed, veterinary and environmental isolates and thereby various types of focus areas of public health authorities. With this approach, relationships between past or international cases could also be inferred using representative public data. We also highlighted the importance and supportive power of epidemiological sample data and an integrated view on both molecular and epidemiological data. Importantly, NGS results still need careful evaluation, as their interpretation approaches often have to be a trade-off between highest resolution and versatility. Standardisation and harmonisation on an international level will further improve using the surplus of information coming from NGS in molecular surveillance.

\section{Acknowledgements}

The authors would like to thank all the colleagues in the public health microbiology and molecular biology laboratories as well as in the food bacteriology laboratories, contributing with excellent laboratory work going beyond their routine diagnostic tasks.

\section{Conflict of interest}

None declared.

Authors' contributions

$A D, A B, U M, R K, S H, N A, A S$ designed the study. UM and $\mathrm{SH}$ provided isolates from culture collections. $A B$ and $U M$ performed and supervised the bacteriology work. AD and RK performed and supervised the molecular analyses. $A D$ analysed the NGS data. $A D$ and $A B$ drafted and revised the 
manuscript. $A D, A B, U M, R K, S H, N A$, AS reviewed and revised the manuscript. $A D$ an $A B$ contributed equally to this article.

\section{References}

1. Rabsch W, Fruth A, Simon S, Szabo I, Malorny B. The zoonotic agent Salmonella. In: Sing A (eds), Zoonoses - Infections Affecting Humans and Animals. Dordrecht: Springer; 2015. p. 179-211. 10/1007978-94-017-9457-2.

2. Koch J, Schrauder A, Alpers K, Werber D, Frank C, Prager R, et al. Salmonella agona outbreak from contaminated aniseed, Germany. Emerg Infect Dis. 2005;11(7):1124-7. https://doi. org/10.3201/eid1107.041022 PMID: 16022796

3. Rabsch W, Prager R, Koch J, Stark K, Roggentin P, Bockemühl J, et al. Molecular epidemiology of Salmonella enterica serovar Agona: characterization of a diffuse outbreak caused by aniseed-fennel-caraway infusion. Epidemiol Infect. 2005;133(5):837-44. https://doi.org/10.1017/ So950268805004152 PMID: 16181503

4. Nicolay N, Thornton L, Cotter S, Garvey P, Bannon O, McKeown $\mathrm{P}$, et al. Salmonella enterica serovar Agona European outbreak associated with a food company. Epidemio Infect. 2011;139(8):1272-80. https://doi.org/10.1017/ So950268810002360 PMID: 20950515

5. Mba-Jonas A, Culpepper W, Hill T, Cantu V, Loera J, Borders J, et al. A Multistate Outbreak of Human Salmonella Agona Infections Associated With Consumption of Fresh, Whole Papayas Imported From Mexico-United States, 2011. Clin Infect Dis. 2018;66(11):1756-61. https://doi.org/10.1093/cid/cix1094 PMID: 29471372

6. Thompson CK, Wang Q, Bag SK, Franklin N, Shadbolt CT, Howard $\mathrm{P}$, et al. Epidemiology and whole genome sequencing of an ongoing point-source Salmonella Agona outbreak associated with sushi consumption in western Sydney, Australia 2015. Epidemiol Infect. 2017;145(10):2062-71. https://doi.org/10.1017/So950268817000693 PMID: 28462733

7. Hörmansdorfer S, Messelhäußer U, Rampp A, Schönberger K, Dallman T, Allerberger F, et al. Re-evaluation of a 2014 multicountry European outbreak of Salmonella Enteritidis phage type $14 \mathrm{~b}$ using recent epidemiological and molecular data. Euro Surveill. 2017;22(50). https://doi.org/10.2807/1560-7917. ES.2017.22.50.17-00196 PMID: 29258650

8. Achtman M, Wain J, Weill FX, Nair S, Zhou Z, Sangal V, et al. S. Enterica MLST Study Group. Multilocus sequence typing as a replacement for serotyping in Salmonella enterica. PLoS Pathog. 2012;8(6):e1002776. https://doi.org/10.1371/journal. ppat.1002776 PMID: 22737074

9. Sandt CH, Krouse DA, Cook CR, Hackman AL, Chmielecki WA, Warren NG. The key role of pulsed-field gel electrophoresis in investigation of a large multiserotype and multistate food-borne outbreak of Salmonella infections centered in Pennsylvania. J Clin Microbiol. 2006;44(9):3208-12. https:// doi.org/10.1128/JCM.01404-06 PMID: 16954249

10. Long SG, DuPont HL, Gaul L, Arafat RR, Selwyn BJ, Rogers J, et al. Pulsed-field gel electrophoresis for Salmonella infection surveillance, Texas, USA, 2007. Emerg Infect Dis. 2010;16(6):983-5. https://doi.org/10.3201/edi1606.091276 PMID: 20507752

11. Piras F, Spanu C, Mocci AM, Demontis M, Santis EPL, Scarano C. Occurrence and traceability of Salmonella spp. in five Sardinian fermented sausage facilities. Ital J Food Saf. 2019;8(1):8011. https://doi.org/10.4081/ijfs.2019.8011 PMID: 31008088

12. van Belkum $A$. The role of short sequence repeats in epidemiologic typing. Curr Opin Microbiol. 1999;2(3):30611. https://doi.org/10.1016/S1369-5274(99)80053-8 PMID: 10383858

13. Lindstedt B-A. Multiple-locus variable number tandem repeats analysis for genetic fingerprinting of pathogenic bacteria. Electrophoresis. 2005;26(13):2567-82. https://doi. org/10.1002/elps.200500096 PMID: 15937984

14. Wuyts V, Mattheus W, De Laminne de Bex G, Wildemauwe C, Roosens NH, Marchal K, et al. MLVA as a tool for public health surveillance of human Salmonella Typhimurium: prospective study in Belgium and evaluation of MLVA loci stability. PLoS One. 2013;8(12):e84055. https://doi.org/10.1371/journal. pone.0084055 PMID: 24391880

15. Vignaud ML, Cherchame E, Marault M, Chaing E, Le Hello S Michel V, et al. MLVA for Salmonella enterica subsp. enterica Serovar Dublin: Development of a Method Suitable for InterLaboratory Surveillance and Application in the Context of a Raw Milk Cheese Outbreak in France in 2012. Front Microbiol. 2017;8:295. https://doi.org/10.3389/fmicb.2017.00295 PMID: 28289408
16. Peters T, Bertrand S, Björkman JT, Brandal LT, Brown DJ, Erdõsi T, et al. Multi-laboratory validation study of multilocus variable-number tandem repeat analysis (MLVA) for Salmonella enterica serovar Enteritidis, 2015. Euro Surveill. 2017;22(9):30477. https://doi.org/10.2807/1560-7917. ES.2017.22.9.30477 PMID: 28277220

17. Malorny B, Junker E, Helmuth R. Multi-locus variable-number tandem repeat analysis for outbreak studies of Salmonella enterica serotype Enteritidis. BMC Microbiol. 2008;8(1):84. https://doi.org/10.1186/1471-2180-8-84 PMID: 18513386

18. European Centre for Disease Prevention and Control (ECDC). Laboratory standard operating procedure for multiple-locus variable-number tandem repeat analysis of Salmonella enterica serotype Enteritidis. Stockholm: ECDC; 2016. https://doi.org/ http://dx.doi.org/10.2900/973540

19. Dallman T, Inns T, Jombart T, Ashton P, Loman N, Chatt C, et al. Phylogenetic structure of European Salmonella Enteritidis outbreak correlates with national and international egg distribution network. Microb Genom. 2016;2(8):e00o070. https://doi.org/10.1099/mgen.0.000070 PMID: 28348865

20. Kanagarajah S, Waldram A, Dolan G, Jenkins C, Ashton PM, Carrion Martin Al, et al. Whole genome sequencing reveals an outbreak of Salmonella Enteritidis associated with reptile feeder mice in the United Kingdom, 2012-2015. Food Microbiol. 2018;71:32-8. https://doi.org/10.1016/j.fm.2017.04.005 PMID: 29366466

21. Zhou Z, McCann A, Litrup E, Murphy R, Cormican M, Fanning $S$, et al. Neutral genomic microevolution of a recently emerged pathogen, Salmonella enterica serovar Agona. PLoS Genet. 2013;9(4):e1003471. https://doi.org/10.1371/journal. pgen.1003471 PMID: 23637636

22. Yoshida CE, Kruczkiewicz P, Laing CR, Lingohr EJ, Gannon VP, Nash JH, et al. The Salmonella In Silico Typing Resource (SISTR): An Open Web-Accessible Tool for Rapidly Typing and Subtyping Draft Salmonella Genome Assemblies. PLoS One. 2016;11(1):e0147101. https://doi.org/10.1371/journal. pone.0147101 PMID: 26800248

23. Jourdan-da Silva N, Fabre L, Robinson E, Fournet N, Nisavanh A, Bruyand M, et al. Ongoing nationwide outbreak of Salmonella Agona associated with internationally distributed infant milk products, France, December 2017. Euro Surveill. 2018;23(2). https://doi.org/10.2807/1560-7917. ES.2018.23.2.17-00852 PMID: 29338811

24. European Food Safety Authority (EFSA) and European Centre for Disease Prevention and Control (ECDC). Multi outbreak of Salmonella Agona infections linked to infant formula. EFSA Supporting Publications.2018;15(1): EN-1365E. https://doi.org/10.2903/sp.efsa.2018.EN-1365

25. Grimont PA, Weill FX. Antigenic Formulae of the Salmonella Serovars, 9th Edition; 2007. Paris: WHO Collaborating Center for Reference and Research on Salmonella.

26. National Center for Biotechnology Information (NCBI). National Center for Biotechnology Information (NCBI) - Sequence Read Archive (SRA). [Accessed Apr 2018]. Available from: https:// www.ncbi.nlm.nih.gov/sra

27. Jünemann S, Sedlazeck FJ, Prior K, Albersmeier A, John U, Kalinowski J, et al. Updating benchtop sequencing performance comparison. Nat Biotechnol. 2013;31(4):294-6. https://doi. org/10.1038/nbt.2522 PMID: 23563421

28. Warwick-Medical-School. Enterobase website - Salmonella database; v1.1.2:Available from: http://enterobase.warwick. ac.uk/species/index/senterica

29. Alikhan NF, Zhou Z, Sergeant MJ, Achtman M. A genomic overview of the population structure of Salmonella. PLoS Genet. 2018;14(4):e1007261. https://doi.org/10.1371/journal. pgen.1007261 PMID: 29621240

30. Ridom GmbH. Ridom SeqSphere+ Documentation - Core Genome MLST Cluster Type. Münster: Ridom GmbH. [Accessed May 2018]. Available from: https://www.ridom.de/seqsphere/ ug/v40/Core_Genome_MLST_Cluster_Type.html

31. Ridom SeqSphere+ version 4.0 User Guide: Available from: https://www.ridom.de/seqsphere/ug/v40/User_Guide.html

32. Kidgell C, Reichard U, Wain J, Linz B, Torpdahl M, Dougan $\mathrm{G}$, et al. Salmonella typhi, the causative agent of typhoid fever, is approximately 50,000 years old. Infect Genet Evol. 2002;2(1):39-45. https://doi.org/10.1016/S15671348(02)00089-8 PMID: 12797999

33. Public Health England (PHE). PHEnix SNP calling pipeline. Copyright 2016. London: PHE. [Accessed Mar 2018]. Available from: https://github.com/phe-bioinformatics/PHEnix

34. Bolger AM, Lohse M, Usadel B. Trimmomatic: a flexible trimmer for Illumina sequence data. Bioinformatics. 2014;30(15):211420. https://doi.org/10.1093/bioinformatics/btu170 PMID: 24695404 
35. Li H, Durbin R. Fast and accurate long-read alignment with Burrows-Wheeler transform. Bioinformatics. 2010;26(5):58995. https://doi.org/10.1093/bioinformatics/btp698 PMID: 20080505

36. McKenna A, Hanna M, Banks E, Sivachenko A, Cibulskis K, Kernytsky A, et al. The Genome Analysis Toolkit: a MapReduce framework for analyzing next-generation DNA sequencing data. Genome Res. 2010;20(9):1297-303. https://doi.org/10.1101/ gr.107524.110 PMID: 20644199

37. Stamatakis A. RAxML-VI-HPC: maximum likelihood-based phylogenetic analyses with thousands of taxa and mixed models. Bioinformatics. 2006;22(21):2688-90. https://doi. org/10.1093/bioinformatics/btl446 PMID: 16928733

38. Pearce ME, Alikhan NF, Dallman TJ, Zhou Z, Grant K, Maiden MCJ. Comparative analysis of core genome MLST and SNP typing within a European Salmonella serovar Enteritidis outbreak. Int J Food Microbiol. 2018;274:1-11. https://doi. org/10.1016/j.ijfoodmicro.2018.02.023 PMID: 29574242

39. Petzold M, Prior K, Moran-Gilad J, Harmsen D, Lück C. Epidemiological information is key when interpreting whole genome sequence data - lessons learned from a large Legionella pneumophila outbreak in Warstein, Germany, 2013. Euro Surveill. 2017;22(45). https://doi.org/10.2807/1560-7917. ES.2017.22.45.17-00137 PMID: 29162202

\section{License, supplementary material and copyright}

This is an open-access article distributed under the terms of the Creative Commons Attribution (CC BY 4.0) Licence. You may share and adapt the material, but must give appropriate credit to the source, provide a link to the licence and indicate if changes were made.

Any supplementary material referenced in the article can be found in the online version.

This article is copyright of the authors or their affiliated institutions, 2019. 